\title{
Core correlation effects in multiconfiguration calculations of isotope shifts in Mg I
}

\author{
Livio Filippin* and Michel Godefroid $^{\dagger}$ \\ Chimie Quantique et Photophysique, Université libre de Bruxelles, B-1050 Brussels, Belgium \\ Jörgen Ekman ${ }^{\ddagger}$ and Per Jönsson ${ }^{\S}$ \\ Group for Materials Science and Applied Mathematics, Malmö University, S-20506 Malmö, Sweden
}

(Received 22 April 2016; published 29 June 2016)

\begin{abstract}
The present work reports results from systematic multiconfiguration Dirac-Hartree-Fock calculations of isotope shifts for several well-known transitions in neutral magnesium. Relativistic normal and specific mass shift factors as well as the electronic probability density at the origin are calculated. Combining these electronic quantities with available nuclear data, energy and transition level shifts are determined for the ${ }^{26} \mathrm{Mg}-{ }^{24} \mathrm{Mg}$ pair of isotopes. Different models for electron correlation are adopted. It is shown that, although valence and core-valence models provide accurate values for the isotope shifts, the inclusion of core-core excitations in the computational strategy significantly improves the accuracy of the transition energies and normal mass shift factors.
\end{abstract}

DOI: 10.1103/PhysRevA.93.062512

\section{INTRODUCTION}

When the effects of the finite mass and the extended spatial charge distribution of the nucleus are taken into account in a Hamiltonian describing an atomic system, the isotopes of an element have different electronic energy levels [1]. The isotope shift (IS) of spectral lines, which consists of the mass shift (MS) and the field shift (FS), plays a key role for extracting the changes in the mean-square charge radius of the atomic nucleus [2-4]. For a given atomic transition $k$ with frequency $v_{k}$, it is assumed that the electronic response of the atom to variations of the nuclear mass and charge distribution can be described by only two factors: the mass shift factor, $\Delta K_{k \text {,MS }}$, and the field shift factor, $F_{k}$, respectively. The observed IS, $\delta v_{k}^{A, A^{\prime}}$, between any pair of isotopes with mass numbers $A$ and $A^{\prime}$ is related to the difference in nuclear masses and in mean-square charge radii, $\delta\left\langle r^{2}\right\rangle^{A, A^{\prime}}[1,2]$.

We perform $a b$ initio calculations of IS electronic factors using the multiconfiguration Dirac-Hartree-Fock (MCDHF) method. This method is implemented in the RIS3 (relativistic isotope shift) module [1], designed for the revised version of the GRASP2K program package [5]. The adopted computational scheme is based on the estimation of the expectation values of the one- and two-body recoil Hamiltonian for a given isotope, including relativistic corrections derived by Shabaev [6,7], combined with the calculation of the total electron densities at the origin. Different correlation models are explored in a systematic way to determine a reliable computational strategy. This strategy is applied on neutral magnesium $(\mathrm{Mg} \mathrm{I})$, which is one of the simplest and best-studied two-valence-electron atoms. As such, it is often used as a test ground for different methods of atomic calculations. In this paper we show that we can accurately calculate the isotope shift of some wellknown transitions in Mg I, where experimental [8-12] and

\footnotetext{
*Livio.Filippin@ulb.ac.be

†mrgodef@ulb.ac.be

†jorgen.ekman@mah.se

§per.jonsson@mah.se
}

theoretical values [13-15] are available for the ${ }^{26} \mathrm{Mg}-{ }^{24} \mathrm{Mg}$ pair of isotopes.

In Sec. II, the principles of the MCDHF method are summarized. In Sec. III, the relativistic expressions of the MS and FS factors are recalled. Section IV enumerates the studied transitions in $\mathrm{Mg}$ I and presents the active space expansion strategy adopted for the electron correlation model. In Sec. V, numerical results of the MS and FS factors are reported for each of the studied transitions, as well as transition energy shifts for the ${ }^{26} \mathrm{Mg}-{ }^{24} \mathrm{Mg}$ pair of isotopes. Section VI reports concluding remarks.

\section{NUMERICAL METHOD}

The MCDHF method [16], as implemented in the GRASP2K program package $[5,17]$, is the fully relativistic counterpart of the nonrelativistic multiconfiguration Hartree-Fock (MCHF) method [18]. The MCDHF method is employed to obtain wave functions that are referred to as atomic state functions (ASFs), i.e., approximate eigenfunctions of the Dirac-Coulomb Hamiltonian given by

$$
\mathcal{H}_{\mathrm{DC}}=\sum_{i=1}^{N}\left[c \boldsymbol{\alpha}_{i} \cdot \boldsymbol{p}_{i}+\left(\beta_{i}-1\right) c^{2}+V\left(r_{i}\right)\right]+\sum_{i<j}^{N} \frac{1}{r_{i j}},
$$

where $V\left(r_{i}\right)$ is the monopole part of the electron-nucleus interaction, $c$ is the speed of light, and $\alpha$ and $\beta$ are the $4 \times 4$ Dirac matrices. An ASF is given as an expansion over $j j$ coupled configuration state functions (CSFs), $\Phi\left(\gamma_{\nu} \Pi J M_{J}\right)$, with the same parity $\Pi$, total angular momentum $J$, and $J_{z}$-projection $M_{J}$ quantum numbers:

$$
\left|\Psi\left(\gamma \Pi J M_{J}\right)\right\rangle=\sum_{\nu=1}^{N_{\mathrm{CSFs}}} c_{\nu}\left|\Phi\left(\gamma_{\nu} \Pi J M_{J}\right)\right\rangle .
$$

In the MCDHF method the radial functions, used to construct the CSFs, and the expansion coefficients $c_{v}$ are determined variationally so as to leave the energy functional

$$
E=\sum_{\mu, v}^{N_{\mathrm{CSFs}}} c_{\mu} c_{\nu}\left\langle\Phi\left(\gamma_{\mu} \Pi J M_{J}\right)\left|\mathcal{H}_{\mathrm{DC}}\right| \Phi\left(\gamma_{\nu} \Pi J M_{J}\right)\right\rangle
$$


stationary with respect to their variations. The resulting coupled radial equations are solved iteratively in the selfconsistent field (SCF) procedure. Once radial functions have been determined, a configuration interaction $(\mathrm{CI})$ calculation is performed over the set of configuration states, providing the expansion coefficients for building the potentials of the next iteration. The SCF and CI coupled processes are repeated until convergence of the total wave function (2) is reached.

\section{ISOTOPE SHIFT THEORY}

The main ideas of the IS theory are outlined here. More details can be found in the works by Shabaev [6,7] and Palmer [19], who pioneered the theory of the relativistic mass shift used in the present work. Gaidamauskas et al. [20] derived the tensorial form of the relativistic recoil operator, implemented in RIS3 [1].

\section{A. Mass shift}

The finite mass of the nucleus gives rise to a recoil effect, called the mass shift (MS). The nuclear recoil corrections within the $(\alpha Z)^{4} \mathrm{~m}^{2} / M$ approximation [6,7] are obtained by evaluating the expectation values of the one- and two-body recoil Hamiltonian for a given isotope,

$$
\mathcal{H}_{\mathrm{MS}}=\frac{1}{2 M} \sum_{i, j}^{N}\left(\boldsymbol{p}_{i} \cdot \boldsymbol{p}_{j}-\frac{\alpha Z}{r_{i}}\left(\boldsymbol{\alpha}_{i}+\frac{\left(\boldsymbol{\alpha}_{i} \cdot \boldsymbol{r}_{i}\right) \boldsymbol{r}_{i}}{r_{i}^{2}}\right) \cdot \boldsymbol{p}_{j}\right),
$$

where $M$ stands for the mass of the nucleus. Separating the one-body $(i=j)$ and two-body $(i \neq j)$ terms that, respectively, constitute the normal mass shift (NMS) and specific mass shift (SMS) contributions, the Hamiltonian (4) can be written

$$
\mathcal{H}_{\mathrm{MS}}=\mathcal{H}_{\mathrm{NMS}}+\mathcal{H}_{\mathrm{SMS}}
$$

The NMS and SMS mass-independent $K$ factors are defined by the following expressions:

$$
K_{\mathrm{NMS}} \equiv M\left\langle\Psi\left|\mathcal{H}_{\mathrm{NMS}}\right| \Psi\right\rangle,
$$

and

$$
K_{\mathrm{SMS}} \equiv M\left\langle\Psi\left|\mathcal{H}_{\mathrm{SMS}}\right| \Psi\right\rangle .
$$

For a transition IS, one needs to consider the variation of the mass shift factor from one level to another. The corresponding line frequency isotope MS between two isotopes, $A$ and $A^{\prime}$, is written as the sum of the NMS and SMS contributions,

$$
\delta v_{k, \mathrm{MS}}^{A, A^{\prime}} \equiv v_{k, \mathrm{MS}}^{A}-v_{k, \mathrm{MS}}^{A^{\prime}}=\delta v_{k, \mathrm{NMS}}^{A, A^{\prime}}+\delta v_{k, \mathrm{SMS}}^{A, A^{\prime}}
$$

with

$$
\delta v_{k, \mathrm{MS}}^{A, A^{\prime}}=\left(\frac{1}{M}-\frac{1}{M^{\prime}}\right) \frac{\Delta K_{k, \mathrm{MS}}}{h}=\left(\frac{1}{M}-\frac{1}{M^{\prime}}\right) \Delta \tilde{K}_{k, \mathrm{MS}} .
$$

Here $\Delta K_{k, \mathrm{MS}}=\left(K_{\mathrm{MS}}^{u}-K_{\mathrm{MS}}^{l}\right)$ is the difference of the $K_{\mathrm{MS}}=$ $K_{\mathrm{NMS}}+K_{\mathrm{SMS}}$ factors of the upper $(u)$ and lower $(l)$ levels involved in the transition $k$. For the $\Delta \tilde{K}$ factors the unit $\mathrm{GHz} \mathrm{u}$ is often used in the literature. As far as the conversion factors are concerned, we use $\Delta K_{k, \mathrm{MS}}\left[m_{e} E_{\mathrm{h}}\right]=$ $3609.4824 \Delta \tilde{K}_{k, \mathrm{MS}}[\mathrm{GHz} \mathrm{u}]$.

\section{B. Field shift}

Neglecting terms of higher order than $\delta\left\langle r^{2}\right\rangle$ in the Seltzer moment [21]

$\lambda^{A, A^{\prime}}=\delta\left\langle r^{2}\right\rangle^{A, A^{\prime}}+b_{1} \delta\left\langle r^{4}\right\rangle^{A, A^{\prime}}+b_{2} \delta\left\langle r^{6}\right\rangle^{A, A^{\prime}}+\cdots$,

the line frequency shift in the transition $k$ arising from the difference in nuclear charge distributions between two isotopes, $A$ and $A^{\prime}$, can be written as [22-24]

$$
\delta v_{k, \mathrm{FS}}^{A, A^{\prime}} \equiv v_{k, \mathrm{FS}}^{A}-v_{k, \mathrm{FS}}^{A^{\prime}}=F_{k} \delta\left\langle r^{2}\right\rangle^{A, A^{\prime}}
$$

In the expression above, $\delta\left\langle r^{2}\right\rangle^{A, A^{\prime}} \equiv\left\langle r^{2}\right\rangle^{A}-\left\langle r^{2}\right\rangle^{A^{\prime}}$, and $F_{k}$ is the line electronic factor given by

$$
F_{k}=\frac{2 \pi}{3 h} Z\left(\frac{e^{2}}{4 \pi \epsilon_{0}}\right) \Delta|\Psi(0)|_{k}^{2},
$$

which is proportional to the change of the total electronic probability density at the origin between level $l$ and level $u$,

$$
\Delta|\Psi(0)|_{k}^{2}=\Delta \rho_{k}^{e}(\mathbf{0})=\rho_{u}^{e}(\mathbf{0})-\rho_{l}^{e}(\mathbf{0})
$$

\section{Total isotope shift}

The total line frequency shift is obtained by merely adding the MS, (8), and FS, (11), contributions:

$$
\begin{aligned}
\delta v_{k}^{A, A^{\prime}} & =\overbrace{\delta v_{k, \mathrm{NMS}}^{A, A^{\prime}}+\delta v_{k, \mathrm{SMS}}^{A, A^{\prime}}}^{\delta v_{k, \mathrm{MS}}^{A, A^{\prime}}}+\delta v_{k, \mathrm{FS}}^{A, A^{\prime}} \\
& =\left(\frac{1}{M}-\frac{1}{M^{\prime}}\right) \Delta \tilde{K}_{k, \mathrm{MS}}+F_{k} \delta\left\langle r^{2}\right\rangle^{A, A^{\prime}} .
\end{aligned}
$$

\section{ACTIVE SPACE EXPANSION}

The transitions in $\mathrm{Mg} \mathrm{I}$ considered in the present work are the following (see Fig. 1): $3 s^{2}{ }^{1} S_{0} \rightarrow 3 s 3 p^{3} P_{1}^{o}$ $(457.2 \mathrm{~nm}), 3 s^{2}{ }^{1} S_{0} \rightarrow 3 s 3 p^{1} P_{1}^{o}(285.3 \mathrm{~nm}), 3 s 3 p^{3} P_{1}^{o} \rightarrow$

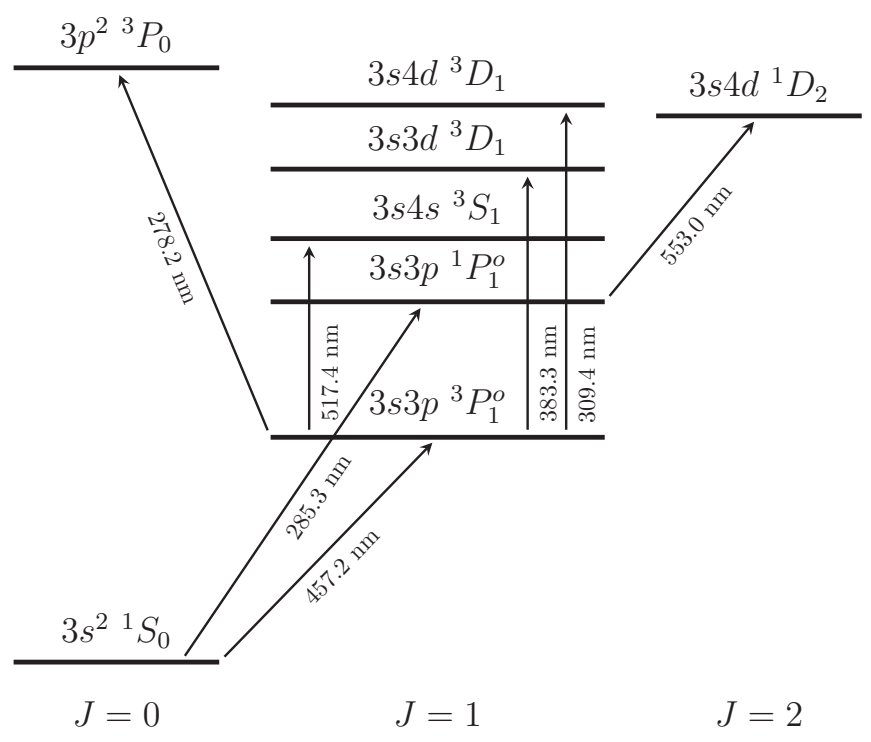

FIG. 1. Schematic diagram of the Mg I transitions. 
$3 s 4 s^{3} S_{1} \quad(517.4 \mathrm{~nm}), \quad 3 s 3 p^{3} P_{1}^{o} \rightarrow 3 p^{23} P_{0} \quad(278.2 \mathrm{~nm})$, $3 s 3 p^{3} P_{1}^{o} \rightarrow 3 s 3 d^{3} D_{1}(383.3 \mathrm{~nm}), 3 s 3 p^{3} P_{1}^{o} \rightarrow 3 s 4 d^{3} D_{1}$ $(309.4 \mathrm{~nm})$, and $3 s 3 p{ }^{1} P_{1}^{o} \rightarrow 3 s 4 d^{1} D_{2}(553.0 \mathrm{~nm})$.

To effectively capture electron correlation, CSFs of a particular symmetry $(J)$ and parity $(\Pi)$ are generated through excitations within an active set of orbitals occupied in the reference configurations and unoccupied virtual orbitals. From hardware and software limitations, it is impossible to use complete active space (CAS) wave functions that would include all CSFs with the appropriate $J$ and $\Pi$ for a given orbital active set. Hence the CSF expansions have to be constrained so that major correlation excitations are taken into account [4].

Single (S) and double (D) substitutions are performed on a multireference (MR) set, which contains the CSFs that have large expansion coefficients and account for the major correlation effects. These SD-MR substitutions take into account valence-valence (VV), core-valence (CV), as well as core-core $(\mathrm{CC})$ correlations. The VV correlation model only allows SD substitutions from valence orbitals, while the $\mathrm{VV}+\mathrm{CV}$ correlation model considers SrD (single and restricted double) substitutions from core and valence orbitals, limiting the excitations to a maximum of one hole in the core. By contrast, the $\mathrm{VV}+\mathrm{CV}+\mathrm{CC}$ correlation model allows all SD substitutions from core and valence orbitals.

Within this approach, a common orbital basis set is chosen for the lower and upper states of each transition. The reference states are obtained using a valence-CAS procedure: SD substitutions are performed within the $n=3,4$ valence orbitals, also including the $5 s$ or both $5 s$ and $6 s$ orbitals in the active space for some transitions (see Table I). The $5 s$ and $6 s$ orbitals are added to account for states belonging to lower configurations with the same $J$ and $\Pi$ in the optimization of the energy functional.

An SCF procedure is then applied to the resulting CSFs, providing the orbital set and the expansion coefficients. Due to limited computer resources, such a valence-CAS multireference set would be too large for subsequent calculations when the active orbital set increases. Hence, for reducing the size of the MR set, only the CSFs whose squared expansion coefficients are larger than a given MR cutoff are kept, i.e., $c_{v}^{2}>\varepsilon_{\mathrm{MR}}$. For each transition, the $\varepsilon_{\mathrm{MR}}$ values and the resulting MR sets are listed in Table I, for the lower and upper states.

The $1 s$ orbital is kept closed in all subsequent calculations; i.e., no substitution from this orbital is allowed. Tests show that opening the $1 s$ orbital does not affect the MS and FS factors to any notable extent. Only orbitals occupied in the singleconfiguration Dirac-Hartree-Fock (DHF) approximation are treated as spectroscopic, and the occupied reference orbitals are frozen in all subsequent calculations. The $J$ levels belonging to a given term are optimized simultaneously with standard weights through the extended optimal level (EOL) scheme [25] and the set of virtual orbitals is increased layer by layer.

For a given transition, the optimization procedure is summarized as follows:

(1) Perform simultaneous calculations for the lower and upper states of the transition, using an MR set consisting of CSFs with the form $2 s^{2} 2 p^{6} n \ln ^{\prime} l^{\prime 2 S+1} L_{J}$ with $n, n^{\prime}=3,4$ $(+5 s$ or $5 s, 6 s)$ and $l, l^{\prime}=s, p, d, f$. Optimize all orbitals simultaneously. These CSFs account for a fair amount of the VV correlation.

(2) Keep the orbitals fixed from step 1, and optimize an orbital basis layer by layer up to $n=8 h$ for both states of the transition, described by CSFs with respective $J^{\Pi}$ symmetries. These CSFs are obtained by SD-MR substitutions with the restriction that there is at most one excitation from the $2 s^{2} 2 p^{6}$ core.

(3) Perform a CI calculation on the CSFs expansion with the $J^{\Pi}$ symmetry of both states, describing $\mathrm{VV}, \mathrm{CV}$, and CC correlation obtained by SD-MR substitutions to the orbital basis from step 2 .

TABLE I. Reference configurations for the lower and upper states of the studied transitions in $\mathrm{Mg}$ I. The MR cutoff values, $\varepsilon_{\mathrm{MR}}$, determine the set of CSFs in the MR space. $N_{\mathrm{CSFs}}$ represents the number of CSFs describing each MR space.

\begin{tabular}{lcccc}
\hline \hline Transition & $\varepsilon_{\mathrm{MR}}$ & $J^{\Pi}$ & & $N_{\mathrm{CSFs}}$ \\
\hline $3 s^{2}{ }^{1} S_{0} \rightarrow 3 s 3 p^{3} P_{1}^{o}$ & 0.01 & $0^{+}$ & {$[\mathrm{Ne}]\left\{3 s^{2}, 3 s 4 s, 3 p^{2}, 3 p 4 p, 3 d^{2}, 4 s^{2}, 4 p^{2}\right\}$} & 11 \\
& & $1^{-}$ & {$[\mathrm{Ne}]\{3 s 3 p, 3 s 4 p, 3 p 3 d, 3 p 4 s, 3 d 4 p, 4 s 4 p\}$} & 14 \\
$3 s^{2}{ }^{1} S_{0} \rightarrow 3 s 3 p{ }^{1} P_{1}^{o}$ & 0.01 & $0^{+}$ & {$[\mathrm{Ne}]\left\{3 s^{2}, 3 s 4 s, 3 p^{2}, 3 p 4 p, 3 d^{2}, 3 d 4 d, 4 s^{2}, 4 p^{2}\right\}$} & 12 \\
& & $1^{-}$ & {$[\mathrm{Ne}]\{3 s 3 p, 3 s 4 p, 3 p 3 d, 3 p 4 s, 3 p 4 d, 3 d 4 p, 3 d 4 f, 4 s 4 p\}$} & 18 \\
$3 s 3 p^{3} P_{1}^{o} \rightarrow 3 s 4 s^{3} S_{1}$ & 0.005 & $1^{-}$ & {$[\mathrm{Ne}]\{3 s 3 p, 3 s 4 p, 3 p 3 d, 3 d 4 p, 3 d 4 f, 4 s 4 p, 3 p 5 s, 4 p 5 s\}$} & 18 \\
& & $1^{+}$ & {$[\mathrm{Ne}]\{3 s 4 s, 3 p 4 p, 3 d 4 d, 3 s 5 s, 4 s 5 s\}$} & 10 \\
$3 s 3 p^{3} P_{1}^{o} \rightarrow 3 p^{2}{ }^{3} P_{0}$ & 0.01 & $1^{-}$ & {$[\mathrm{Ne}]\{3 s 3 p, 3 s 4 p, 3 p 3 d, 3 d 4 p, 4 s 4 p\}$} & 19 \\
& & $0^{+}$ & {$[\mathrm{Ne}]\left\{3 s^{2}, 3 s 4 s, 3 s 5 s, 3 s 6 s, 3 p^{2}, 3 p 4 p, 3 d^{2}, 4 s^{2}, 4 s 5 s, 4 s 6 s, 4 p^{2}, 4 f^{2}, 5 s 6 s, 5 s^{2}, 6 s^{2}\right\}$} \\
$3 s 3 p^{3} P_{1}^{o} \rightarrow 3 s 3 d^{3} D_{1}$ & 0.01 & $1^{-}$ & {$[\mathrm{Ne}]\{3 s 3 p, 3 p 3 d, 3 p 4 s, 3 p 4 d, 3 d 4 p, 4 s 4 p, 4 p 4 d\}$} & 9 \\
& & $1^{+}$ & {$[\mathrm{Ne}]\{3 s 3 d, 3 p 4 p, 3 p 4 f, 3 d 4 s, 4 s 4 d, 4 p 4 f\}$} & 19 \\
$3 s 3 p^{3} P_{1}^{o} \rightarrow 3 s 4 d^{3} D_{1}$ & 0.01 & $1^{-}$ & {$[\mathrm{Ne}]\{3 s 3 p, 3 p 3 d, 3 p 4 d, 3 d 4 p, 3 d 4 f, 4 s 4 p, 4 p 4 d, 3 p 5 s, 3 p 6 s, 4 p 6 s\}$} & 23 \\
& & $1^{+}$ & {$[\mathrm{Ne}]\left\{3 s 3 d, 3 s 4 s, 3 s 4 d, 3 s 5 s, 3 p 4 p, 3 p 4 f, 3 d 4 s, 3 d 4 d, 3 p^{2}, 3 d^{2}, 4 s 4 d, 4 s 5 s, 4 p 4 f, 4 p^{2}\right.$,} & 23 \\
$3 s 3 p{ }^{1} P_{1}^{o} \rightarrow 3 s 4 d^{1} D_{2}$ & 0.025 & $1^{-}$ & {$[\mathrm{Ne}]\{3 s 3 p, 3 s 4 p, 3 p 3 d, 3 p 4 s, 3 p 4 d, 3 d 4 p\}$} & 12 \\
& & $2^{+}$ & {$[\mathrm{Ne}]\left\{3 s 3 d, 3 s 4 d, 3 p^{2}, 3 p 4 p, 3 p 4 f\right\}$} & 11 \\
\hline \hline
\end{tabular}


(4) Keep the orbitals up to $8 h$ fixed from step 2 and optimize one additional layer of orbitals using CC substitutions from the $\mathrm{Mg}^{2+} 2 s^{2} 2 p^{6}(Z=12)$ core. The orbitals of this additional layer target $\mathrm{CC}$ correlation and are therefore contracted.

(5) Perform a CI calculation on the CSFs expansion with the $J^{\Pi}$ symmetry of both states of the transition, describing $\mathrm{VV}, \mathrm{CV}$, and CC correlation obtained by SD-MR substitutions to the orbital basis from step 4 ( $n=8 h+$ the additional layer).

Following the procedure in steps 1 and 2 or steps $1-5$, respectively, yields results labeled "CV" or "CC" in Tables III and IV.

The CC effects are more balanced with a common orbital basis for describing both upper and lower states, resulting in more accurate transition energies, as mentioned in Ref. [13].

The CSF expansions become significantly large when CC correlations are taken into account, counting up to $2 \times 10^{6}$ CSFs. Hence, applying an SCF procedure to such an amount of CSFs takes too much computing time. This justifies the use of the CI method at that stage.

The effect of adding the Breit interaction to the DiracCoulomb Hamiltonian, (1), is found to be much smaller than the uncertainty in the transition IS factors with respect to the correlation model. This interaction has therefore been neglected in the procedure.

\section{NUMERICAL RESULTS}

In this section, MS and FS electronic factors, $\Delta \tilde{K}_{k, \mathrm{MS}}$ and $F_{k}$, as well as total IS, $\delta v_{k}^{26,24}$, given by

$$
\delta v_{k}^{26,24}=\left(\frac{1}{M_{26}}-\frac{1}{M_{24}}\right) \Delta \tilde{K}_{k, \mathrm{MS}}+F_{k} \delta\left\langle r^{2}\right\rangle^{26,24}
$$

of the ${ }^{26} \mathrm{Mg}-{ }^{24} \mathrm{Mg}$ pair of isotopes are calculated for the studied transitions in $\mathrm{Mg}$ I.

Nuclear masses $(M)$ are calculated by subtracting the mass of the electrons from the atomic mass $\left(M_{\text {atom }}\right)$, and by adding the binding energy $\left(B_{\mathrm{el}}\right)$, using the formula

$$
M(A, Z)=M_{\text {atom }}(A, Z)-Z m_{e}+B_{\mathrm{el}}(Z),
$$

where the total electronic binding energy (in eV) is estimated using [26,27]

$$
B_{\mathrm{el}}(Z)=14.4381 Z^{2.39}+1.55468 \times 10^{-6} Z^{5.35} .
$$

Atomic masses are provided in Ref. [28]. The resulting values of the nuclear masses are respectively

$$
M_{26}=25.97601589 \mathrm{u}
$$

and

$$
M_{24}=23.97846462 \mathrm{u} \text {. }
$$

The NMS factor, $\Delta \tilde{K}_{k, \text { NMS }}$, can be approximated through the scaling law

$$
\Delta \tilde{K}_{k, \mathrm{NMS}} \approx-m_{e} \nu_{k}^{\mathrm{expt}},
$$

where $m_{e}$ is the mass of the electron and $v_{k}^{\text {expt }}$ is the experimental transition energy of transition $k$, available in the NIST database [29]. The transition NMS is then deduced from Eq. (20) using expressions (8) and (9), i.e.,

$$
\delta v_{k, \mathrm{NMS}}^{26,24} \approx\left(\frac{m_{e}}{M_{24}}-\frac{m_{e}}{M_{26}}\right) v_{k}^{\mathrm{expt}} .
$$

The reliability of the FS values obtained with the $a b$ initio electronic $F_{k}$ factor, (12), is a function of the accuracy of the calculations, but also of the level of confidence on the nuclear data $\delta\left\langle r^{2}\right\rangle^{A, A^{\prime}}$. Values compiled by Angeli and Marinova [30] provide the mean-square charge radii difference between ${ }^{26} \mathrm{Mg}$ and ${ }^{24} \mathrm{Mg}$ :

$$
\delta\left\langle r^{2}\right\rangle^{26,24} \equiv\left\langle r^{2}\right\rangle^{26}-\left\langle r^{2}\right\rangle^{24}=-0.1419 \mathrm{fm}^{2} .
$$

Let us first study the convergence of the level MS factors, $K_{\mathrm{NMS}}$ and $K_{\mathrm{SMS}}$ (in $m_{e} E_{\mathrm{h}}$ ), and the electronic probability density at the origin, $\rho^{e}(\mathbf{0})$ (in $a_{0}^{-3}$ ), of a given transition as a function of the increasing active space. Table II displays the values for the $3 s^{2}{ }^{1} S_{0} \rightarrow 3 s 3 p{ }^{1} P_{1}^{o}$ transition, with an MR cutoff $\varepsilon_{\mathrm{MR}}$ equal to 0.01 . Within each correlation model, the active space is extended until convergence of the differential

\begin{tabular}{|c|c|c|c|c|c|c|c|c|c|c|}
\hline \multirow[b]{2}{*}{ Active space } & \multirow[b]{2}{*}{ Notation } & \multicolumn{3}{|c|}{$K_{\mathrm{NMS}}\left(m_{e} E_{\mathrm{h}}\right)$} & \multicolumn{3}{|c|}{$K_{\mathrm{SMS}}\left(m_{e} E_{\mathrm{h}}\right)$} & \multicolumn{3}{|c|}{$\rho^{e}(\mathbf{0})\left(a_{0}^{-3}\right)$} \\
\hline & & Lower & Upper & $\Delta_{l}^{u}$ & Lower & Upper & $\Delta_{l}^{u}$ & Lower & Upper & $\Delta_{l}^{u}$ \\
\hline \multicolumn{11}{|l|}{ VV model } \\
\hline $4 s 4 p 4 d 4 f$ & $\operatorname{VV} 4 f$ & 199.6791 & 199.4764 & -0.2027 & -27.5167 & -27.4115 & 0.1052 & 1157.2594 & 1156.4404 & -0.8190 \\
\hline \multicolumn{11}{|l|}{$\mathrm{VV}+\mathrm{CV}$ model } \\
\hline $5 s 5 p 5 d 5 f 5 g$ & $\mathrm{CV} 5 g$ & 199.6023 & 199.4798 & -0.1225 & -27.3690 & -27.3370 & 0.0320 & 1157.5654 & 1156.5885 & -0.9769 \\
\hline $6 s 6 p 6 d 6 f 6 g 6 h$ & $\mathrm{CV} 6 h$ & 199.6306 & 199.4951 & -0.1355 & -27.3645 & -27.3266 & 0.0379 & 1157.6171 & 1156.6106 & -1.0065 \\
\hline $7 s 7 p 7 d 7 f 7 g 7 h$ & $\mathrm{CV} 7 h$ & 199.6337 & 199.4974 & -0.1363 & -27.3600 & -27.3288 & 0.0312 & 1157.6394 & 1156.6151 & -1.0243 \\
\hline $8 s 8 p 8 d 8 f 8 g 8 h$ & $\mathrm{CV} 8 h$ & 199.6338 & 199.4974 & -0.1364 & -27.3518 & -27.3239 & 0.0279 & 1157.6481 & 1156.6246 & -1.0235 \\
\hline \multicolumn{11}{|l|}{$\mathrm{VV}+\mathrm{CV}+\mathrm{CC}$ model } \\
\hline $8 s 8 p 8 d 8 f 8 g 8 h$ & $\mathrm{CC} 8 h$ & 199.9113 & 199.7546 & -0.1567 & -24.3327 & -24.2797 & 0.0530 & 1157.6349 & 1156.6514 & -0.9835 \\
\hline $9 s 9 p 9 d 9 f 9 g 9 h$ & CC $9 h$ & 199.9401 & 199.7829 & -0.1572 & -24.3200 & -24.2668 & 0.0532 & 1157.6521 & 1156.6665 & -0.9856 \\
\hline
\end{tabular}
results $\Delta_{l}^{u}$ is obtained.

For $K_{\mathrm{NMS}}$, adding the $n=5$ layer of orbitals optimized on $\mathrm{VV}$ and CV correlations (denoted as "CV $5 g$ " in Table II) slightly shifts the value for the lower level, $3 s^{2}{ }^{1} S_{0}$, while

TABLE II. Level MS factors, $K_{\mathrm{NMS}}$ and $K_{\mathrm{SMS}}$ (in $m_{e} E_{\mathrm{h}}$ ), and the electronic probability density at the origin, $\rho^{e}(\mathbf{0})$ (in $a_{0}^{-3}$ ), as functions of the increasing active space for the $3 s^{2}{ }^{1} S_{0} \rightarrow 3 s 3 p^{1} P_{1}^{o}$ transition in Mg I. $\Delta_{l}^{u}$ stands for the difference between the values of the upper level and the lower level. Results are obtained with an MR cutoff $\varepsilon_{\mathrm{MR}}=0.01$. 
the value for the upper level, $3 s 3 p^{1} P_{1}^{o}$, remains nearly constant. However, this small variation leads to a significant modification $(40 \%)$ of the differential value, $\Delta K_{\mathrm{NMS}}$. The convergence of the results is achieved by adding the successive layers within the $\mathrm{VV}+\mathrm{CV}$ model, when the active space includes the $n=8$ correlation layer (denoted as " $\mathrm{CV} 8 h$ "). Adding CC correlations through the CI computation described in step 3 of Sec. IV (denoted as "CC 8h") shifts both level values and hence does not drastically modify $\Delta K_{\mathrm{NMS}}$ $(15 \%)$. The convergence is obtained for $\Delta K_{\mathrm{NMS}}$ within the $\mathrm{VV}+\mathrm{CV}+\mathrm{CC}$ model, with the procedure of steps 4 and 5 (denoted as "CC $9 h$ ").

The situation is different for $K_{\mathrm{SMS}}$. Adding the $n=5$ layer in the active space ( $\mathrm{CV} 5 g$ ) modifies both level and differential values. The convergence for $\Delta K_{\text {SMS }}$ within the $\mathrm{VV}+\mathrm{CV}$ model is slower than for $\Delta K_{\mathrm{NMS}}$. It is only obtained when the $n=9$ correlation layer is included, where $\Delta K_{\mathrm{SMS}}=$ $0.0275 m_{e} E_{\mathrm{h}}$. It is indeed well known that the SMS factor is more sensitive to correlation effects than the NMS factor, as expected from the two-body nature of the SMS operator. However, the inclusion of this last $\mathrm{VV}+\mathrm{CV}$ correlation layer does not affect the results when $\mathrm{CC}$ correlations are added and hence is not considered in this work. The procedure of step 3 (CC $8 h$ ) leads to a drastic change in the level values, and also in the $\Delta K_{\mathrm{SMS}}$ value $(90 \%)$. Within the $\mathrm{VV}+\mathrm{CV}+\mathrm{CC}$ model, the procedure of steps 4 and 5 (CC $9 h$ ) leads to the convergence of $\Delta K_{\mathrm{SMS}}$.

The convergence is smoother for $\rho^{e}(\mathbf{0})$ compared with $K_{\mathrm{SMS}}$, as expected from the one-body nature of the density operator, like the NMS operator. The $\Delta \rho^{e}(\mathbf{0})$ value converges within the $\mathrm{VV}+\mathrm{CV}$ model $(\mathrm{CV} 8 h)$. Adding CC correlations in step 3 (CC $8 h)$ does not significantly affect both level and differential values. Within the $\mathrm{VV}+\mathrm{CV}+\mathrm{CC}$ model, the procedure of steps 4 and 5 ( $\mathrm{CC} 9 h$ ) leads to the convergence of $\Delta \rho^{e}(\mathbf{0})$.

A look at both the MS and FS factors displayed in Table II shows that small variations in the level values due to correlation effects can lead to a significant variation in the differential values, $\Delta_{l}^{u}$. This illustrates how sensitive these electronic factors are, and hence how challenging it is to obtain reliable values with such a computational approach. This observation is general for all other transitions studied in this work.

Let us now study the impact of the MR cutoff $\varepsilon_{\text {MR }}$ value, i.e., the size of the MR set, on the accuracy of the transition

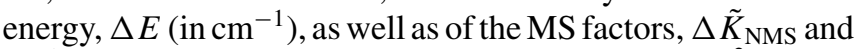
$\Delta \tilde{K}_{\mathrm{SMS}}$ (in $\mathrm{GHz} \mathrm{u}$ ), and the FS factor, $F$ (in $\mathrm{MHz} / \mathrm{fm}^{2}$ ). Fig. 2 displays the convergence plots for the $3 s^{2}{ }^{1} S_{0} \rightarrow 3 s 3 p{ }^{1} P_{1}^{o}$ transition, as a function of the increasing active space. Two $\varepsilon_{\mathrm{MR}}$ values are considered: 0.05 (dashed lines) and 0.01 (solid lines). For $\varepsilon_{\mathrm{MR}}=0.01$, the $\mathrm{MR}$ set of both upper and lower states of this transition is given in Table I, and the MS and FS results (given in other units) are displayed in Table II. For $\varepsilon_{\mathrm{MR}}=0.05$, the reference configurations are $[\mathrm{Ne}]\left\{3 s^{2}, 3 p^{2}, 3 p 4 p\right\}$ for the lower state (five CSFs) and $[\mathrm{Ne}]\{3 s 3 p, 3 s 4 p, 3 p 3 d, 3 p 4 s, 3 d 4 p\}$ for the upper state (nine CSFs). The size of these MR sets is thus much smaller. The results of $\Delta E$ are compared with the NIST Atomic Spectra Database values [29], while those of $\Delta \tilde{K}_{\mathrm{NMS}}$ and $\Delta \tilde{K}_{\mathrm{SMS}}$ are respectively compared with the scaling law (20) and with benchmark values from Berengut et al. [15], in excellent agreement with observation (see Table IV). These reference values are represented by straight lines in Fig. 2 .

Within the VV model (VV $4 f$ ), the values using $\varepsilon_{\mathrm{MR}}=$ 0.05 and 0.01 are the same for each property. Indeed, the computation is performed on the full set of CSFs, before selecting two $\varepsilon_{\mathrm{MR}}$ values leading to different MR sets. Within the VV+CV model (from CV $5 g$ to $\mathrm{CV} 8 h$ ), the behavior of both lines is nearly the same.

Significant differences occur when $\mathrm{CC}$ correlations are added (CC $8 h$ and $\mathrm{CC} 9 h)$. The $\mathrm{CC} 9 h$ value of $\Delta E$ is $35179 \mathrm{~cm}^{-1}$ with $\varepsilon_{\mathrm{MR}}=0.05$ and $35063 \mathrm{~cm}^{-1}$ with $\varepsilon_{\mathrm{MR}}=$ 0.01 , which is closer to the NIST value of $35051 \mathrm{~cm}^{-1}$. The same observation holds for $\Delta \tilde{K}_{\mathrm{NMS}}$. The CC $9 h$ value is $-661 \mathrm{GHz} u$ with $\varepsilon_{\mathrm{MR}}=0.05$ and $-566 \mathrm{GHz} \mathrm{u}$ with $\varepsilon_{\mathrm{MR}}=$ 0.01 , the latter being closer to the scaling law result of $-576 \mathrm{GHz}$ u. Equation (20), although only strictly valid in the nonrelativistic framework, is used as a reference value since the relativistic effects are expected to be small for $Z=12$. The relativistic corrections to $\Delta \tilde{K}_{\mathrm{NMS}}$ can be deduced with RIS3 by computing the expectation values of the nonrelativistic part of the recoil Hamiltonian (4), which provides $-576 \mathrm{GHz} \mathrm{u}$, reproducing the scaling law result. The relativistic corrections are thus rather small $(2 \%)$.

The situation is different for $\Delta \tilde{K}_{\mathrm{SMS}}$. The $\mathrm{CC} 9 h$ value with $\varepsilon_{\mathrm{MR}}=0.01(192 \mathrm{GHz} \mathrm{u})$ is slightly higher than the one with $\varepsilon_{\mathrm{MR}}=0.05(178 \mathrm{GHz} \mathrm{u})$. To discriminate between the two results, they are compared with the values from Berengut et al. [15]. In Ref. [15], $\Delta \tilde{K}_{\mathrm{NMS}}$ is evaluated with the scaling law (20), and $\Delta \tilde{K}_{\text {SMS }}$ is obtained by the finite-field scaling method. In this technique, the rescaled nonrelativistic SMS operator is added to the relativistic many-particle Hamiltonian

$$
\mathcal{H}_{\lambda}=\mathcal{H}_{0}+\lambda \mathcal{H}_{\mathrm{SMS}}=\mathcal{H}_{0}+\lambda \sum_{i<j} \boldsymbol{p}_{i} \cdot \boldsymbol{p}_{j}
$$

The eigenvalue problem for Hamiltonian (23) is solved for various $\lambda$ using a combination of the CI method and manybody perturbation theory (MBPT). Then the level $K_{\mathrm{SMS}}$ factor is evaluated as

$$
K_{\mathrm{SMS}}=\lim _{\lambda \rightarrow 0} \frac{d E}{d \lambda} .
$$

The value of $\Delta \tilde{K}_{\mathrm{SMS}}$ provided by Ref. [15] is $134 \mathrm{GHz} \mathrm{u}$, closer to the result obtained with the higher MR cutoff. This illustrates again the challenge of providing reliable values of $\Delta \tilde{K}_{\text {SMS }}$. For the SMS factor, the relativistic corrections are small $(2 \%)$, as expected.

For the FS factor, $F$, the addition of CC correlations leads to two different values at the $\mathrm{CC} 9 h$ stage: $-58 \mathrm{MHz} / \mathrm{fm}^{2}$ for $\varepsilon_{\mathrm{MR}}=0.01$, against $-63 \mathrm{MHz} / \mathrm{fm}^{2}$ for $\varepsilon_{\mathrm{MR}}=0.05$. Their relative difference can be used to provide an upper bound of the uncertainty on the $F$ factor, equal to $8 \%$. This value can be further used in a King plot technique, as the uncertainty on the slope of the straight line, for instance.

The same computation has been performed with an extended MR set. It led to the conclusion that lowering the value of $\varepsilon_{\mathrm{MR}}$ beyond 0.01 does not improve the accuracy of the results. The obtained values with $\varepsilon_{\mathrm{MR}}=0.01$ are thus stable with respect to supplementary correlation effects in the computational procedure. This property holds for all the other transitions studied in this work. 

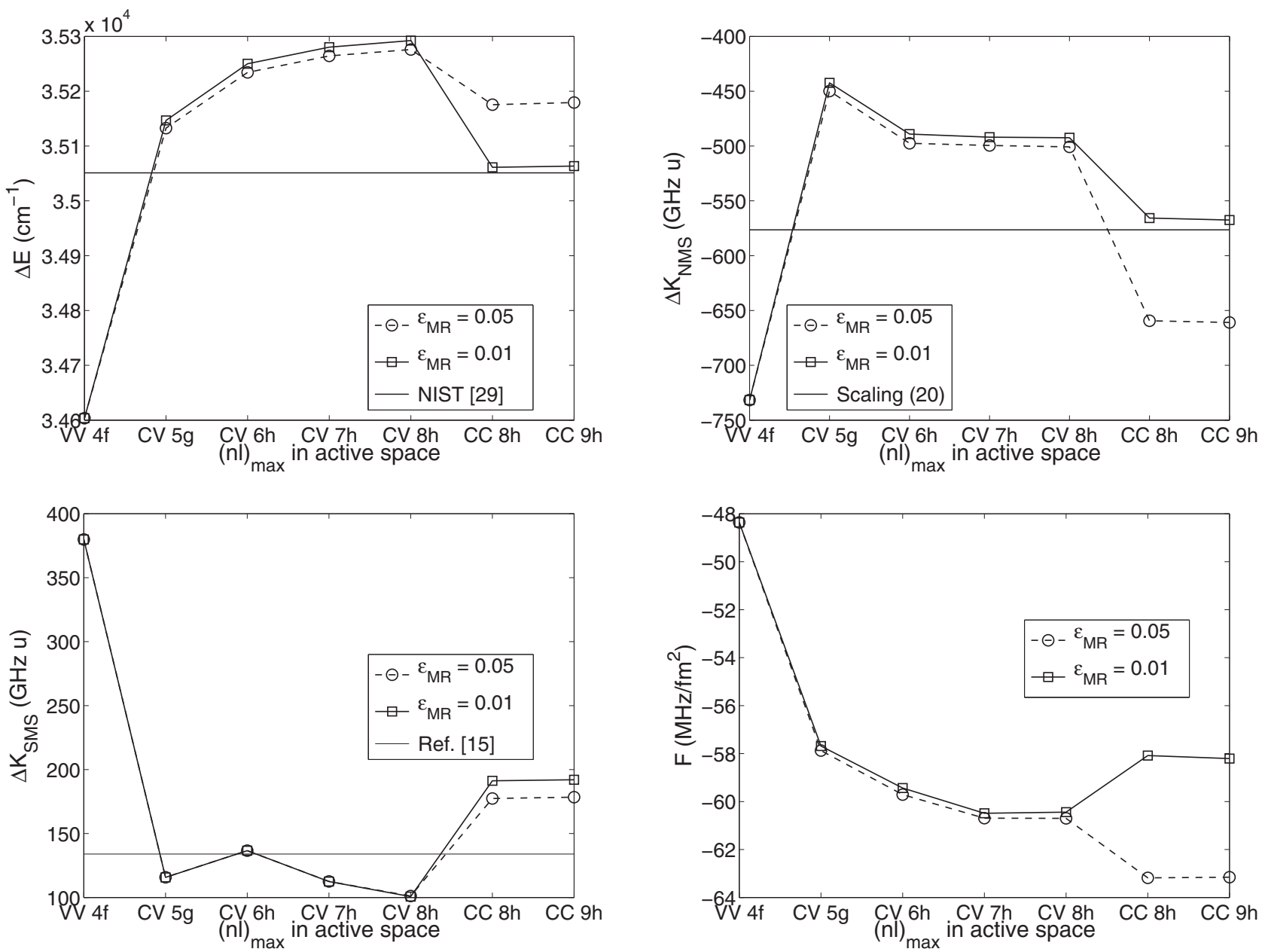

FIG. 2. Transition energy, $\Delta E$ (in $\mathrm{cm}^{-1}$ ), MS factors, $\Delta \tilde{K}_{\mathrm{NMS}}$ and $\Delta \tilde{K}_{\mathrm{SMS}}$ (in GHz u), and FS factor, $F$ (in MHz/fm ${ }^{2}$ ), as functions of the increasing active space for the $3 s^{2}{ }^{1} S_{0} \rightarrow 3 s 3 p{ }^{1} S_{1}^{o}$ transition in $\mathrm{Mg}$ I. The correlation models are labeled VV, CV, and CC, and $(n l)_{\text {max }}$ denotes the maximal $n$ and $l$ values of the orbitals in the active set. Results are obtained with two MR cutoff values $\varepsilon_{\text {MR }}: 0.05$ (dashed lines) and 0.01 (solid lines). Comparison of $\Delta E$ with the NIST value [29], of $\Delta \tilde{K}_{\mathrm{NMS}}$ with the scaling law from Eq. (20), and of $\Delta \tilde{K}_{\mathrm{SMS}}$ with the benchmark value from Ref. [15].

A common observation of the plots displayed in Fig. 2 shows that, although the convergence of the properties is reached within the $\mathrm{VV}+\mathrm{CV}$ model, the obtained values at that stage are not in excellent agreement with experimental

data. This emphasizes the need to include CC excitations in the computational procedure in order to provide more accurate results. This observation is also general for all other transitions studied in this work.

TABLE III. Transition energies, $\Delta E$ (in $\mathrm{cm}^{-1}$ ), MS factors, $\Delta \tilde{K}_{\mathrm{NMS}}$ and $\Delta \tilde{K}_{\mathrm{SMS}}$ (in GHz u), and FS factors, $F$ (in $\mathrm{MHz} / \mathrm{fm}^{2}$ ), of the studied transitions in Mg I. Comparison of $\Delta E$ with values from the NIST database [29] and theoretical results [15]. $\Delta \tilde{K}_{\mathrm{NMS}}$ and $\Delta \tilde{K}_{\mathrm{SMS}}$ are respectively compared with values from the scaling law (20) (Scal.) and with values from Ref. [15].

\begin{tabular}{|c|c|c|c|c|c|c|c|c|c|c|c|c|}
\hline \multirow[b]{2}{*}{ Transition } & \multicolumn{4}{|c|}{$\Delta E\left(\mathrm{~cm}^{-1}\right)$} & \multicolumn{3}{|c|}{$\Delta \tilde{K}_{\mathrm{NMS}}(\mathrm{GHz} \mathrm{u})$} & \multicolumn{3}{|c|}{$\Delta \tilde{K}_{\mathrm{SMS}}(\mathrm{GHz} \mathrm{u})$} & \multicolumn{2}{|c|}{$F\left(\mathrm{MHz} / \mathrm{fm}^{2}\right)$} \\
\hline & $\mathrm{CV}$ & $\mathrm{CC}$ & NIST [29] & Ref. [15] & $\mathrm{CV}$ & $\mathrm{CC}$ & Scal. (20) & $\mathrm{CV}$ & $\mathrm{CC}$ & Ref. [15] & $\mathrm{CV}$ & $\mathrm{CC}$ \\
\hline $3 s^{2}{ }^{1} S_{0} \rightarrow 3 s 3 p^{3} P_{1}^{o}$ & 21970 & 21780 & 21870 & 21794 & -277 & -354 & -360 & -544 & -417 & -491 & -77 & -73 \\
\hline $3 s^{2}{ }^{1} S_{0} \rightarrow 3 s 3 p{ }^{1} P_{1}^{o}$ & 35292 & 35063 & 35051 & 35050 & -492 & -567 & -576 & 101 & 192 & 134 & -60 & -58 \\
\hline $3 s 3 p^{3} P_{1}^{o} \rightarrow 3 s 4 s^{3} S_{1}$ & 19474 & 19311 & 19327 & 19332 & -325 & -315 & -318 & 453 & 416 & 442 & 40 & 39 \\
\hline $3 s 3 p^{3} P_{1}^{o} \rightarrow 3 p^{2}{ }^{3} P_{0}$ & 36084 & 35857 & 35943 & 35912 & -501 & -570 & -591 & -17 & 97 & 27 & -100 & -95 \\
\hline $3 s 3 p^{3} P_{1}^{o} \rightarrow 3 s 3 d^{3} D_{1}$ & 26324 & 26069 & 26087 & 26085 & -421 & -443 & -429 & 408 & 403 & 414 & 26 & 24 \\
\hline $3 s 3 p^{3} P_{1}^{o} \rightarrow 3 s 4 d^{3} D_{1}$ & 32535 & 32173 & 32322 & 32317 & -504 & -505 & -532 & 402 & 415 & 403 & 29 & 27 \\
\hline $3 s 3 p^{1} P_{1}^{o} \rightarrow 3 s 4 d^{1} D_{2}$ & 18245 & 17882 & 18084 & 17987 & -251 & -269 & -297 & -412 & -319 & -373 & -5 & -7 \\
\hline
\end{tabular}


TABLE IV. Total IS, NMS, SMS, and FS (in MHz), between ${ }^{26} \mathrm{Mg}$ and ${ }^{24} \mathrm{Mg}$ of the studied transitions in Mg I. Comparison of NMS with values from the scaling law (21) (Scal.). Comparison of IS and SMS with values extracted from experiments and theoretical results [15], where the FS contribution is ignored (20-30 MHz).

\begin{tabular}{|c|c|c|c|c|c|c|c|c|c|c|c|c|c|}
\hline \multirow[b]{2}{*}{ Transition } & \multicolumn{4}{|c|}{ IS (MHz) } & \multicolumn{3}{|c|}{ NMS (MHz) } & \multicolumn{4}{|c|}{ SMS (MHz) } & \multicolumn{2}{|c|}{ FS (MHz) } \\
\hline & $\mathrm{CV}$ & $\mathrm{CC}$ & Expt. & Ref. [15] & $\mathrm{CV}$ & $\mathrm{CC}$ & Scal. (21) & $\mathrm{CV}$ & $\mathrm{CC}$ & Expt. & Ref. [15] & $\mathrm{CV}$ & $\mathrm{CC}$ \\
\hline $3 s^{21} S_{0} \rightarrow 3 s 3 p^{3} P_{1}^{o}$ & 2643 & 2482 & $2683(0)^{\mathrm{a}}$ & 2726 & 888 & 1135 & 1153 & 1744 & 1337 & $1530^{\mathrm{a}}$ & 1573 & 11 & 10 \\
\hline $3 s^{2}{ }^{1} S_{0} \rightarrow 3 s 3 p{ }^{1} P_{1}^{o}$ & 1262 & 1210 & $1414(8)^{\mathrm{b}}$ & 1420 & 1577 & 1814 & 1848 & -324 & -612 & $-434^{\mathrm{b}}$ & -428 & 9 & 8 \\
\hline $3 s 3 p^{3} P_{1}^{o} \rightarrow 3 s 4 s^{3} S_{1}$ & -418 & -330 & $-390(5)^{\mathrm{c}}$ & -397 & 1041 & 1009 & 1019 & -1453 & -1333 & $-1409^{c}$ & -1416 & -6 & -6 \\
\hline $3 s 3 p^{3} P_{1}^{o} \rightarrow 3 p^{23} P_{0}$ & 1674 & 1529 & $1810(80)^{\mathrm{d}}$ & 1809 & 1606 & 1827 & 1895 & 54 & -311 & $-85^{\mathrm{d}}$ & -86 & 14 & 13 \\
\hline $3 s 3 p^{3} P_{1}^{o} \rightarrow 3 s 3 d^{3} D_{1}$ & 37 & 125 & $61(3)^{\mathrm{e}}$ & 49 & 1349 & 1420 & 1375 & -1308 & -1292 & $-1314^{\mathrm{e}}$ & -1326 & -4 & -3 \\
\hline $3 s 3 p^{3} P_{1}^{o} \rightarrow 3 s 4 d^{3} D_{1}$ & 324 & 287 & $420(20)^{\mathrm{d}}$ & 413 & 1616 & 1620 & 1704 & -1288 & -1329 & $-1284^{\mathrm{d}}$ & -1291 & -4 & -4 \\
\hline $3 s 3 p^{1} P_{1}^{o} \rightarrow 3 s 4 d^{1} D_{2}$ & 2124 & 1883 & $2107(15)^{\mathrm{c}}$ & 2148 & 804 & 862 & 953 & 1321 & 1022 & $1154^{\mathrm{c}}$ & 1195 & 1 & 1 \\
\hline
\end{tabular}

${ }^{\mathrm{a}}$ Reference [8].

${ }^{\mathrm{b}}$ Reference [9].

${ }^{\mathrm{c} R e f e r e n c e ~[10] . ~}$

${ }^{\mathrm{d}}$ Reference [11].

${ }^{\mathrm{e}}$ Reference [12].

Table III displays the transition energies, $\Delta E\left(\mathrm{in} \mathrm{cm}^{-1}\right), \mathrm{MS}$ factors, $\Delta \tilde{K}_{\mathrm{NMS}}$ and $\Delta \tilde{K}_{\mathrm{SMS}}$ (in $\mathrm{GHz} \mathrm{u}$ ), and FS factors, $F$ (in $\mathrm{MHz} / \mathrm{fm}^{2}$ ), of the studied transitions in $\mathrm{Mg}$ I. As mentioned in Sec. IV, the labels $\mathrm{CV}$ and $\mathrm{CC}$ respectively correspond to the computational procedure in steps 1 and 2 or in steps $1-5$.

The values of $\Delta E$ are compared with NIST data [29] and benchmark results from Berengut et al. [15]. The correction brought by the inclusion of CC correlations is clear. At the $\mathrm{CV}$ stage all transition energies are overestimated. In contrast, at the CC stage they decrease and become very close to the NIST values. The relative error lies between $0.03 \%$ for the $3 s^{2}{ }^{1} S_{0} \rightarrow$ $3 s 3 p^{1} P_{1}^{o}$ and $1.12 \%$ for the $3 s 3 p^{1} P_{1}^{o} \rightarrow 3 s 4 d^{1} D_{2}$ transition, while the calculation performed in Ref. [15] provides relative errors within $0.4 \%$ for all considered transitions. The same observation holds for $\Delta \tilde{K}_{\text {NMS }}$. The values are overestimated at the CV stage and become very close to the scaling law results (21) at the CC stage.

Similarly to the study of the $3 s^{2}{ }^{1} S_{0} \rightarrow 3 s 3 p{ }^{1} P_{1}^{o}$ transition in Fig. 2, the results of $\Delta \tilde{K}_{\mathrm{SMS}}$ for the other transitions at the CC stage are not in better agreement with Ref. [15] than the one obtained at the CV stage. They are even less accurate for all considered transitions. These differences represent the major source of discrepancies between this work and experimental values of total IS in Mg I, as highlighted in Table IV.

By contrast, the value of the $F$ factor is not significantly affected by the addition of CC correlations. It varies by a few $\mathrm{MHz} / \mathrm{fm}^{2}$ from the $\mathrm{CV}$ to the $\mathrm{CC}$ stage.

Table IV displays the values of the total IS, NMS, SMS, and FS (in MHz) between ${ }^{26} \mathrm{Mg}$ and ${ }^{24} \mathrm{Mg}$ of the studied transitions in $\mathrm{Mg}$ I. The NMS and SMS contributions are obtained by multiplying $\Delta \tilde{K}_{\mathrm{NMS}}$ and $\Delta \tilde{K}_{\mathrm{SMS}}$ by the factor $\left(1 / M_{26}-\right.$ $\left.1 / M_{24}\right)$, using Eqs. (18) and (19). The FS contributions are obtained by multiplying $F$ by $\delta\left\langle r^{2}\right\rangle^{26,24}$, using Eq. (22). The total IS is given by Eq. (15).

The conclusions for the isotope parameters above also hold for the NMS, SMS, and FS energies, since they are obtained by multiplying the corresponding electronic factors displayed in Table III by nuclear constants. The NMS results are compared with the scaling law values from Eq. (21), while the SMS results are compared with values extracted from experiments [8-12] and theoretical results of Ref. [15]. The FS contribution is ignored in Ref. [15] for simplicity, since the authors found it to be approximately 20-30 MHz. Indeed, the FS value is less than the experimental uncertainty in most transitions and is of the order of the error in their SMS calculations. The present results agree with the order of magnitude, but the range of values for the FS is found to be from -6 to $+14 \mathrm{MHz}$ instead.

When considering the total IS, it is worth observing that the $\mathrm{CV}$ values are in better agreement with observation than the $\mathrm{CC}$ ones, for all the studied transitions. Indeed, the errors made on both NMS and SMS within the VV+CV model seem to "accidentally" cancel, providing more accurate values for the total IS. By contrast, within the $\mathrm{VV}+\mathrm{CV}+\mathrm{CC}$ model the NMS values are closer to the scaling law results, but the SMS values are not improved in comparison. Summing up NMS and SMS leads thus to less accurate results for the total IS.

Compared to the values from Ref. [15], the total IS is in less good agreement with observation for all studied transitions, whether CC correlations are included or not. Indeed, the MBPT+CI method is known to be the most accurate computational technique for one- and two-valence-electron atoms. Nevertheless, these results show that CC effects need to be accounted for in the computational strategy in order to improve the values of $\Delta E$ and $\Delta \tilde{K}_{\mathrm{NMS}}$ for each of the studied transitions in $\mathrm{Mg} \mathrm{I}$.

\section{CONCLUSION}

The present work describes an ab initio method for the relativistic calculation of the IS in many-electron atoms using the MCDHF approach. The accuracy of the computational procedure is tested by estimating the energy shifts of the ${ }^{26} \mathrm{Mg}-{ }^{24} \mathrm{Mg}$ pair of isotopes, for several well-known transitions in $\mathrm{Mg}$ I.

Different models for electron correlation are adopted. Within each model, the convergence of the level MS factors and the electronic probability density at the origin, as a function 
of the increasing active space, is studied for the $3 s^{2}{ }^{1} S_{0} \rightarrow$ $3 s 3 p^{1} P_{1}^{o}$ transition. It is shown that small variations in the level values due to correlation effects can lead to a significant variation in the differential values, highlighting the challenge in providing accurate results for the SMS factors with this computational approach. The impact of the MR cutoff value on the accuracy of the transition energy and the MS and FS electronic factors is investigated as a function of the increasing active space, for the same transition. It leads to the conclusion that extending the MR set beyond a certain MR cutoff value does not improve the accuracy of the results.

The study of the electronic factors for other transitions in $\mathrm{Mg}$ I shows that CC correlation needs to be accounted for in the computational strategy in order to obtain accurate values for the transition energies and the NMS factors. The convergence of the results when including an additional orbital layer optimized on $\mathrm{CC}$ substitutions from the $\mathrm{Mg}^{2+}$ core is highly satisfactory. By contrast, in comparison with benchmark calculations from Berengut et al. [15], the accuracy of the SMS factor values is not improved when $\mathrm{CC}$ contributions are added.

Total IS, NMS, SMS, and FS are computed between ${ }^{26} \mathrm{Mg}$ and ${ }^{24} \mathrm{Mg}$ for the studied transitions in $\mathrm{Mg}$ I. The agreement of the numerical results is found to be good for all transitions. It is surprisingly better for the $\mathrm{VV}+\mathrm{CV}$ model, although the transition energies and the NMS factors are less accurate than in the $\mathrm{VV}+\mathrm{CV}+\mathrm{CC}$ model. In the former, the errors made on NMS and SMS cancel each other out "accidentally," providing more accurate values for the total IS. Nevertheless, for both correlation models, the present accuracy is in particular high enough for the purposes of resolving systematic errors in the search for the fine-structure constant variation, and for studies of the isotopic evolution of the universe [15].

A possible way to improve the accuracy of the results is the use of the partitioned correlation function interaction (PCFI) approach [31]. It is based on the idea of relaxing the orthonormality restriction on the orbital basis, and breaking down the very large calculations in the traditional multiconfiguration methods into a series of smaller parallel calculations. This method is very flexible for targeting different electron correlation effects. CC effects in IS factors could be then treated more accurately and efficiently with the use of this technique. Additionally, electron correlation effects beyond the SD-MR model (such as triple and quadruple excitations) can be included perturbatively. Work is being done in these directions.

\section{ACKNOWLEDGMENTS}

This work has been partially supported by the Belgian F.R.S.-FNRS Fonds de la Recherche Scientifique (CDR J.0047.16) and the BriX IAP Research Program No. P7/12 (Belgium). L.F. acknowledges the support from the FRIA. J.E. and P.J. acknowledge financial support from the Swedish Research Council (VR), under Contract No. 2015-04842.
[1] C. Nazé, E. Gaidamauskas, G. Gaigalas, M. Godefroid, and P. Jönsson, Comput. Phys. Commun. 184, 2187 (2013).

[2] W. H. King, Isotope Shifts in Atomic Spectra (Plenum Press, New York, 1984).

[3] B. Cheal, T. E. Cocolios, and S. Fritzsche, Phys. Rev. A 86, 042501 (2012).

[4] C. Nazé, J. G. Li, and M. Godefroid, Phys. Rev. A 91, 032511 (2015).

[5] P. Jönsson, G. Gaigalas, J. Bieron, C. Froese Fischer, and I. P. Grant, Comput. Phys. Commun. 184, 2197 (2013).

[6] V. M. Shabaev, Theor. Math. Phys. 63, 588 (1985).

[7] V. M. Shabaev, Yad. Fiz. 47, 107 (1988) [Sov. J. Nucl. Phys. 47, 69 (1988)].

[8] U. Sterr, K. Sengstock, J. H. Müller, and W. Ertmer, Appl. Phys. B: Photophys. Laser Chem. 56, 62 (1993).

[9] E. J. Salumbides, S. Hannemann, K. S. E. Eikema, and W. Ubachs, Mon. Not. R. Astron. Soc. 373, L41 (2006).

[10] L. Hallstadius and J. E. Hansen, Z. Phys. A 285, 365 (1978).

[11] C. Novero, A. Godone, and G. M. Tino, Nuovo Cimento Soc. Ital. Fis. D 14, 955 (1992).

[12] L. Hallstadius, Z. Phys. A 291, 203 (1979).

[13] L. Veseth, J. Phys. B 20, 235 (1987).

[14] P. Jönsson, C. Froese Fischer, and M. R. Godefroid, J. Phys. B 32, 1233 (1999).

[15] J. C. Berengut, V. V. Flambaum, and M. G. Kozlov, Phys. Rev. A 72, 044501 (2005).

[16] I. P. Grant, Relativistic Quantum Theory of Atoms and Molecules (Springer, New York, 2007).

[17] P. Jönsson, X. He, C. Froese Fischer, and I. P. Grant, Comput. Phys. Commun. 177, 597 (2007).
[18] C. Froese Fischer, G. Tachiev, G. Gaigalas, and M. R. Godefroid, Comput. Phys. Commun. 176, 559 (2007).

[19] C. Palmer, J. Phys. B 21, 1951 (1988).

[20] E. Gaidamauskas, C. Nazé, P. Rynkun, G. Gaigalas, and P. Jönsson, J. Phys. B 44, 175003 (2011).

[21] E. C. Seltzer, Phys. Rev. 188, 1916 (1969).

[22] G. Fricke, C. Bernhardt, K. Heilig, L. Schaller, L. Schellenberg, E. Shera, and C. De Jager, At. Data Nucl. Data Tables 60, 177 (1995).

[23] G. Torbohm, B. Fricke, and A. Rosén, Phys. Rev. A 31, 2038 (1985).

[24] S. Blundell, P. Baird, C. Palmer, D. Stacey, and G. Woodgate, J. Phys. B 20, 3663 (1987).

[25] K. G. Dyall, I. P. Grant, C. T. Johnson, F. A. Parpia, and E. P. Plummer, Comput. Phys. Commun. 55, 425 (1989).

[26] K. N. Huang, M. Aoyagi, M. Chen, and B. Crasemann, At. Data Nucl. Data Tables 18, 243 (1976).

[27] D. Lunney, J. M. Pearson, and C. Thibault, Rev. Mod. Phys. 75, 1021 (2003).

[28] J. S. Coursey, D. J. Schwab, J. J. Tsai, and R. A. Dragoset, Atomic Weights and Isotopic Compositions (Version 3.0) (NIST, Gaithersburg, MD, 2012), http://physics.nist.gov/Comp

[29] A. Kramida, Y. Ralchenko, and J. Reader, NIST Atomic Spectra Database (Version 5.3) (NIST, Gaithersburg, MD, 2015), http://physics.nist.gov/asd

[30] I. Angeli and K. Marinova, At. Data Nucl. Data Tables 99, 69 (2013).

[31] S. Verdebout, P. Rynkun, P. Jönsson, G. Gaigalas, C. Froese Fischer, and M. Godefroid, J. Phys. B 46, 085003 (2013). 